\title{
Influência da cristalização na dureza, módulo de elasticidade e tenacidade à fratura por indentação em vitrocerâmicas de dissilicato de lítio $\left(\mathrm{Li}_{2} \mathrm{O} .2 \mathrm{SiO}_{2}\right)$
}

\section{(Influence of crystallization on hardness, elastic modulus and fracture toughness in lithium disilicate $\mathrm{Li}_{2} \mathrm{O} .2 \mathrm{SiO}_{2}$ glass-ceramics)}

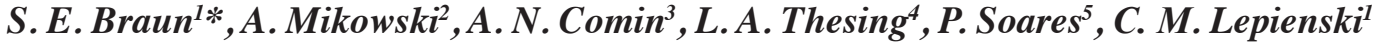 \\ ${ }^{1}$ Universidade Federal do Paraná, Departamento de Física, PR, Brasil \\ ${ }^{2}$ Universidade Federal de Santa Catarina, Departamento de Engenharias da Mobilidade, SC, Brasil \\ ${ }^{3}$ Universidade Federal de Viçosa, Departamento de Engenharia Agrícola, MG, Brasil \\ ${ }^{4}$ Universidade Federal do Pampa, Departamento de Engenharia Mecânica, RS, Brasil \\ ${ }^{5}$ Pontifícia Universidade Católica do Paraná, Departamento de Engenharia Mecânica, PR, Brasil
}

\begin{abstract}
Resumo
O efeito da cristalização sobre as propriedades mecânicas (dureza, módulo de elasticidade e tenacidade à fratura por indentação) do vitrocerâmico dissilicato de lítio $\mathrm{Li}_{2} \mathrm{O} .2 \mathrm{SiO}_{2}$ foi investigado pela técnica de indentação (indentação instrumentada, microdurezas Vickers e Knoop). Os tratamentos térmicos para cristalização foram realizados em duas etapas: nucleação em $475^{\circ} \mathrm{C}$ por 2,5 e $10 \mathrm{~h}$, e crescimento de cristais em $563{ }^{\circ} \mathrm{C}$ por $0,5,1,2,3$ e $24 \mathrm{~h}$. Foram determinadas as frações cristalizadas, que foram correlacionadas com a dureza, o módulo de elasticidade e a tenacidade à fratura por indentação. Os testes de microdureza foram realizados utilizandose penetradores Vickers e Knoop. O módulo de elasticidade das regiões amorfas e cristalinas foi medido por testes de indentação instrumentada com um indentador Berkovich, realizado em cada fase separadamente. A modificação da morfologia das trincas geradas por indentação com pontas piramidais foi avaliada para amostras com diferentes frações cristalinas utilizando microscopia óptica. Foi discutida a alteração dos padrões de trincas, para diferentes graus de cristalização, na forma de determinação da tenacidade à fratura por indentação. Os maiores valores de dureza e tenacidade foram obtidos em amostras com 100\% de fração cristalina, sendo que maiores tempos de nucleação de cristais levaram a melhores valores nas propriedades mecânicas investigadas. Os valores obtidos para amostras $100 \%$ de fração cristalina foram: tenacidade à fratura por indentação de 1,8 MPa.m², microdureza Knoop de 7,2 GPa e módulo de elasticidade de $155 \mathrm{GPa}$.

Palavras-chave: tenacidade à fratura por indentação, microdureza, módulo de elasticidade, indentação Vickers, vitrocerâmica, dissilicato de lítio.
\end{abstract}

\begin{abstract}
The effect of crystallization on the mechanical properties (hardness, modulus of elasticity and fracture toughness) of lithium disilicate $\mathrm{Li}_{2} \mathrm{O} .2 \mathrm{SiO}_{2}$ glass-ceramic was investigated by indentation (instrumented indentation, and Vickers and Knoop microhardness). The heat treatments for crystallization were carried out in two stages: nucleation at $475{ }^{\circ} \mathrm{C}$ for 2,5 and $10 \mathrm{~h}$, and crystals growth at $563{ }^{\circ} \mathrm{C}$ for 0.5, 1, 2, 3 and $24 \mathrm{~h}$. Crystal fractions were correlated to hardness, elastic modulus and fracture toughness. Hardness was measured by Knoop and Vickers indenters. The modulus of elasticity of the amorphous and crystalline regions was measured by instrumented indentation tests with a Berkovich indenter, performed in each phase separately. The morphology of cracks generated by indentation with pyramid indenters was investigated for samples with different crystalline fractions using optical microscopy. The highest values of hardness and toughness were obtained in samples with $100 \%$ crystalline fraction, with the highest nucleation time. The obtained values were: fracture toughness of $1.8 \mathrm{MPa} \cdot \mathrm{m}^{1 / 2}$, Knoop microhardness of $7.2 \mathrm{GPa}$ and elastic modulus of $155 \mathrm{GPa}$.
\end{abstract}

Keywords: fracture toughness, microhardness, elastic modulus, Vickers indentation, glass-ceramic, lithium disilicate.

\section{INTRODUÇÃO}

Os vidros são considerados materiais frágeis e os defeitos existentes na superfície são precursores de trincas, resultando em baixa resistência mecânica. Os defeitos podem surgir devido à exposição dos vidros à umidade atmosférica ou a danos mecânicos [1]. Assim, os tratamentos térmicos que promovem a cristalização constituemse em eficientes métodos para aumentar a resistência mecânica desses materiais frágeis. $\mathrm{O}$ tratamento térmico 
para cristalização pode deixar a superfície do material vitrocerâmico sob compressão após resfriamento. Se o coeficiente de expansão térmica da camada cristalina for menor que o da matriz vítrea, conseguem-se propriedades mecânicas superiores aos dos vidros convencionais. No tratamento térmico para cristalização podem atuar também mecanismos de aprisionamento e eliminação de trincas superficiais, em consequência da alteração da superfície e dos defeitos gerados pelo crescimento dos cristais. $\mathrm{Na}$ maioria dos casos, o cristal possui dureza maior do que o vidro, o que torna o material cristalino menos susceptível ao aparecimento de defeitos durante seu uso, obtendo-se, assim, um acréscimo no tempo de vida útil do material vitrocerâmico [2]. Neste trabalho, utilizaram-se técnicas de indentação para se determinar o efeito da cristalização nas propriedades mecânicas (dureza, módulo de elasticidade e tenacidade à fratura por indentação) de materiais vitrocerâmicos com a composição do dissilicato de lítio $\mathrm{Li}_{2} \mathrm{O} \cdot 2 \mathrm{SiO}_{2}$. Entre os métodos mais utilizados na avaliação da tenacidade à fratura por indentação destacam-se os métodos de flexão em quatro pontos, de um corpo de prova pré-entalhado e de impressão por indentação Vickers [3]. $\mathrm{O}$ método de indentação Vickers tem sido muito utilizado para estimar a tenacidade à fratura por indentação $\left(\mathrm{K}_{\mathrm{C}}\right)$ de vidros e vitrocerâmicas. Os vários métodos existentes diferem na abordagem matemática, mas são essencialmente baseados na medição do comprimento de trincas geradas durante a indentação. Uma lista de métodos de indentação pode ser obtida em $[4,5]$, bem como a comparação com outros métodos convencionais de mecânica da fratura. As vantagens do método de indentação incluem o tamanho pequeno da amostra de teste, a facilidade da preparação da amostra e a simplicidade do teste [6]. No entanto, como apontado em [7], este método induz a erros para materiais que apresentam mecanismos de endurecimento por cristalização do vidro. O controle da cristalização em vitrocerâmicas envolve dois estágios de tratamento conhecidos como estágio de nucleação e o estágio de cristalização. No estágio de nucleação pequenos núcleos são formados dentro da matriz vítrea. Após a formação do núcleo estável, a cristalização ocorre por crescimento de uma nova fase cristalina [8].

Os exemplos de aumento da resistência mecânica devido à cristalização do vidro são muito descritos na literatura. Foi observado um aumento de tenacidade à fratura por indentação por um fator quatro após a cristalização em vidros no sistema $\mathrm{CaO}-\mathrm{MgO}-\mathrm{SiO}_{2}-\mathrm{P}_{2} \mathrm{O}_{5}$ [9]. A tenacidade à fratura por indentação também foi observada aumentar com o aumento da fração cristalina em vitrocerâmicas no sistema $\mathrm{SiO}_{2}-\mathrm{Al}_{2} \mathrm{O}_{3}-\mathrm{P}_{2} \mathrm{O}_{5}-\mathrm{CaO}-\mathrm{CaF}_{2}$. $\mathrm{O}$ aumento de resistência é devido à geração de tensões compressivas permanentes na superfície da matriz de vidro e modificações nas propriedades mecânicas [10]. Foi investigada a relação entre a resistência e o tamanho do cristal para o sistema de vitrocerâmica $\mathrm{LiO}_{2} \cdot \mathrm{SiO}_{2}$. A geração de tensões compressivas elevadas na superfície requer a produção de cristais com um coeficiente de expansão térmica menor que o do vidro. Cristais muito pequenos não afetam significativamente a propagação de trincas, pois, como as mesmas se propagam mais facilmente ao longo de planos de clivagem, em grãos pequenos a mudança de direção da propagação da trinca é menor que em grãos maiores. Os vidros apresentaram tenacidade à fratura por indentação, dureza e módulo de elasticidades inferiores aos obtidos para as vitrocerâmicas [11]. Em vitrocerâmicas no sistema $\mathrm{CaO}-\mathrm{Al}_{2} \mathrm{O}_{3}-\mathrm{SiO}_{2}$, a orientação cristalina causada pela adição de $\mathrm{Y}_{2} \mathrm{O}_{3}$ resultou em aumento de dureza de 84 para $110 \mathrm{MPa}$, com $3,25 \%$ de $\mathrm{Y}_{2} \mathrm{O}_{3}$ [12]. Foi verificado que as trincas geralmente não se propagam a partir dos cantos da impressão. Tais trincas claramente propagam-se ao longo dos planos preferenciais de clivagem e a tenacidade à fratura por indentação de $\mathrm{SiC}$ é comparativamente mais baixa ao longo destes planos. A tenacidade à fratura por indentação obtida foi $1,84 \mathrm{MPa} \cdot \mathrm{m}^{1 / 2}$ no caso das trincas se propagarem na direção dos planos preferenciais de clivagem, sendo esse valor mais baixo que o valor de 3,46 MPa.m ${ }^{1 / 2}$ quando as trincas ocorriam na mesma direção que a diagonal da impressão [13]. Em trabalhos mais recentes há outros exemplos. Uma vitrocerâmica à base de dissilicato de lítio para aplicações dentárias apresentou resistência à flexão de $439 \pm 93 \mathrm{MPa}$ após tratamento térmico a $675{ }^{\circ} \mathrm{C}$ [14]. Um método de sinterização de reação combinada com tecnologia de prensagem a quente foi desenvolvido para preparar vitrocerâmicas a partir de vidro $\mathrm{Li}_{2} \mathrm{Si}_{2} \mathrm{O}_{5}$ com alta resistência à flexão, dureza e tenacidade à fratura por indentação [15]. A resistência à flexão aumentou rapidamente com a cristalização em frações de volume de cristal abaixo de $10 \%$ e atingiu um valor 2,5 vezes maior que o do vidro original [16]. Em outro trabalho, os autores examinaram o comportamento da propagação de trincas de três vitrocerâmicas submetidos à indentação Vickers e observaram que existe uma forte relação entre a microestrutura da vitrocerâmica, o caminho da trinca e a resistência do material [17]. O tratamento a altas temperaturas e pressões no vidro de dissilicato de lítio melhora as propriedades mecânicas [18]. O comportamento mecânico do metassilicato de lítio vitrocerâmico foi investigado usando nanoindentação e técnicas de imagem com sonda de varredura in situ. A dureza de contato, Hc, e o módulo de Young, E, foram medidos com carga de pico de $10 \mathrm{mN}$ a uma taxa de aplicação de 0,1 a $2 \mathrm{mN} / \mathrm{s}$ com o objetivo de avaliar o efeito da taxa de aplicação da carga nas suas propriedades. A dureza de contato aumentou com a taxa de carregamento [19]. O efeito da alta pressão sobre o vidro dissilicato de lítio com composição estequiométrica $\mathrm{Li}_{2} \mathrm{O} .2 \mathrm{SiO}_{2}$ no ambiente químico $\mathrm{Si} 2 \mathrm{p}, \mathrm{O} 1 \mathrm{~s}$ e Li 1 s foi investigado por espectroscopia fotoelétrica de raios $\mathrm{X}$. Foi verificado que a amostra cristalizada a 7,7 $\mathrm{GPa}$ indicou a formação de metassilicato de lítio e $\mathrm{SiO}_{2}$ [20]. Durante o processo de cristalização surgem tensões residuais em torno de cristais devido ao desajuste entre o coeficiente de expansão térmica das fases vítrea e cristalina [21-24]. Estes campos de tensões trativas e compressivas afetam a propagação de trincas e devem ser considerados. 
O objetivo do presente trabalho foi avaliar as propriedades mecânicas de vitrocerâmicas à base de dissilicato de lítio, nas quais foram medidos os níveis de cristalização, os quais foram correlacionados com a dureza, o módulo de elasticidade e a tenacidade à fratura por indentação. A partir disso, foram traçados os seguintes objetivos específicos: verificar as fases cristalinas para correlacionar com as propriedades mecânicas; investigar os valores de dureza e módulo de elasticidade por nanoindentação; comparar a dureza Knoop e Vickers; verificar a dependência da carga de indentação sobre os valores de dureza; determinar os valores de tenacidade à fratura por indentação com o aumento da fração cristalina superficial em relação ao vidro dissilicato de lítio, sem tratamento térmico; estudar a fractografia para investigar os padrões de trincas gerados em função do grau de cristalização para os diferentes tratamentos térmicos. A evolução dos padrões de trinca foi utilizada para analisar a variação da tenacidade à fratura por indentação. Para este estudo, escolheu-se o sistema dissilicato de lítio, um dos sistemas vitrocerâmicos mais investigados. Além disso, é um dos poucos sistemas em que a cristalização ocorre homogeneamente no volume, permitindo um controle de microestrutura, isto é, um número e tamanho de cristais por controle cuidadoso do tempo e da temperatura de tratamento térmico. $\mathrm{O}$ dissilicato de lítio $\left(\mathrm{Li}_{2} \mathrm{O} .2 \mathrm{SiO}_{2}\right)$ possui vários aspectos interessantes. Constitui um dos mais importantes sistemas vitrocerâmicos, pois foi extensamente investigado, possuindo excelente durabilidade térmica e física, além disso, cristaliza-se mais facilmente que outros vidros alcalinos servindo como modelo para o estudo de sistemas vitrocerâmicos mais complexos. Como vitrocerâmica, é utilizada comercialmente, por exemplo, em próteses dentárias devido à sua boa biocompatibilidade, resistência à compressão, condutividade térmica semelhante à dos tecidos dentais, estabilidade de cor e, principalmente, elevado potencial para simular a aparência dos dentes.

\section{MATERIAIS E MÉTODOS}

Materiais: as amostras utilizadas foram obtidas de vidros de dissilicato de lítio fornecidos pelo Laboratório de Materiais Vítreos (LaMaV) da Universidade Federal de São Carlos. O vidro dissilicato de lítio $\left(\mathrm{Li}_{2} \mathrm{O} .2 \mathrm{SiO}_{2}\right)$ obtido com a composição molar nominal estequiométrica $\left(33,3 \%\right.$ mol $\left.\mathrm{Li}_{2} \mathrm{O}\right)$ foi preparado usando carbonato de lítio $\left(\mathrm{Li}_{2} \mathrm{CO}_{3}\right) 99 \%$ e sílica com alto teor de pureza (>99,9\%). As fusões foram realizadas em um forno elétrico a 1450 ${ }^{\circ} \mathrm{C}$, em cadinhos de platina. Os fundidos foram então vertidos, moídos e refundidos à mesma temperatura. Esses procedimentos foram repetidos resultando num vidro em forma de barra visualmente homogêneo e sem bolhas.

Preparação das amostras: a preparação das amostras envolveu as seguintes etapas: corte das amostras, lixamento (desbaste) e polimento (acabamento). Foi utilizado corte por abrasão, utilizando-se um disco impregnado por pó abrasivo de diamante. $\mathrm{O}$ disco foi adaptado a uma cortadeira elétrica de precisão adequada ao corte de amostras de espessura reduzida. Todas as amostras foram cortadas com espessura de aproximadamente $3 \mathrm{~mm}$. O lixamento foi realizado utilizando lixas de carbeto de silício em série decrescente no tamanho de granulometria variando de 200 até 1200 (ANSI B74.18-1996). O acabamento superficial foi realizado por polimento mecânico. No polimento mecânico, o acabamento da superfície ocorre devido à ação abrasiva de partículas mais finas que as utilizadas nas lixas, dispersas sobre um pano. A politriz utilizada na preparação das amostras foi da Panambra, DP-10. Os panos de polimento utilizados foram da Buehler. O agente de polimento utilizado foi óxido de cério em pó, misturado com água potável.

Tratamentos térmicos: os tratamentos térmicos duplos foram escolhidos por possibilitarem gerar reduzido número de cristais com tamanhos grandes. As amostras foram nucleadas a $475{ }^{\circ} \mathrm{C}$ durante $5 \mathrm{~h}$, depois cristalizadas a $563{ }^{\circ} \mathrm{C}$ durante períodos de $0,5,1,2,3$ e $24 \mathrm{~h}$ (completamente cristalino) no forno elétrico tubular com controle de temperatura de $\pm 1{ }^{\circ} \mathrm{C}$. As superfícies cristalinas de vitrocerâmica foram removidas por lixamento subsequente com lixa de $\mathrm{SiC}$ e polimento com óxido de cério antes dos ensaios de indentação, para eliminar a camada cristalina de superfície.

Caracterização das amostras: a fração cristalina foi determinada pela área relativa da fase cristalina e amorfa através de microscopia óptica e software de análise de imagem (BX-51, Olympus). O comprimento e a largura dos cristais foram determinados utilizando-se o programa Image J. Propriedades mecânicas: a dureza e a tenacidade à fratura por indentação foram medidas utilizando-se um indentador Vickers (HVS-1000, Fisher), com cargas aplicadas de 5,10 e $20 \mathrm{~N}$ a um tempo de permanência de 30 s. Foi obtida uma média de trinta indentações para cada amostra em cada carga. A dureza foi determinada a partir da área de impressão enquanto que a tenacidade à fratura por indentação foi determinada a partir do comprimento de trincas radiais por microscopia óptica. O módulo de elasticidade das regiões amorfas e cristalinas foi medido por testes de indentação instrumentada com um indentador Berkovich, realizado em cada fase separadamente (Nanoindenter XP, MTS). O valor correto do módulo de elasticidade é uma das fontes de erro associada à fratura gerada por um indentador Vickers. Na literatura [25] foi possível encontrar estudos comparando algumas equações, onde foram obtidos valores de tenacidade à fratura por indentação $\left(\mathrm{K}_{\mathrm{C}}\right)$ de um material de referência com valor de $\mathrm{K}_{\mathrm{C}}$ a partir das equações de Niiihara, Anstis e Miyoshi. Os autores concluíram que o valor obtido a partir da equação de Miyoshi foi o que mais se aproximou do valor de referência. Em seguida, para um material cerâmico SiC- $\alpha$, os valores obtidos a partir destas três equações foram comparados com a faixa de valores obtida por técnicas padrões de medida de $K_{C}$. A equação que obteve os resultados mais próximos dos valores obtidos pelas técnicas padrões foi a de Anstis [25]. Neste trabalho, o cálculo de tenacidade à 
fratura por indentação foi realizado a partir da equação de Anstis:

$$
\mathrm{K}_{\mathrm{c}}=\chi\left(\frac{\mathrm{E}}{\mathrm{HV}}\right)^{\frac{1}{2}} \frac{\mathrm{P}}{\mathrm{c}^{\frac{3}{2}}}
$$

em que E é o módulo de elasticidade, obtido normalmente pelo método Oliver [26], HV é a dureza Vickers, P é a carga aplicada, c é o comprimento da trinca radial e $\chi$ é uma constante empírica igual a $0,016 \pm 0,004$ [27].

\section{RESULTADOS E DISCUSSÃO}

Dureza e módulo de elasticidade medidas por nanoindentação: a Fig. 1 mostra os gráficos da variação da dureza e módulo de elasticidade em função do tempo de tratamento térmico de crescimento de cristal. Na Fig. 2 são apresentadas as imagens de microscopia óptica das duas diferentes matrizes de indentações realizadas em torno de diferentes cristais para amostras com tratamentos térmicos

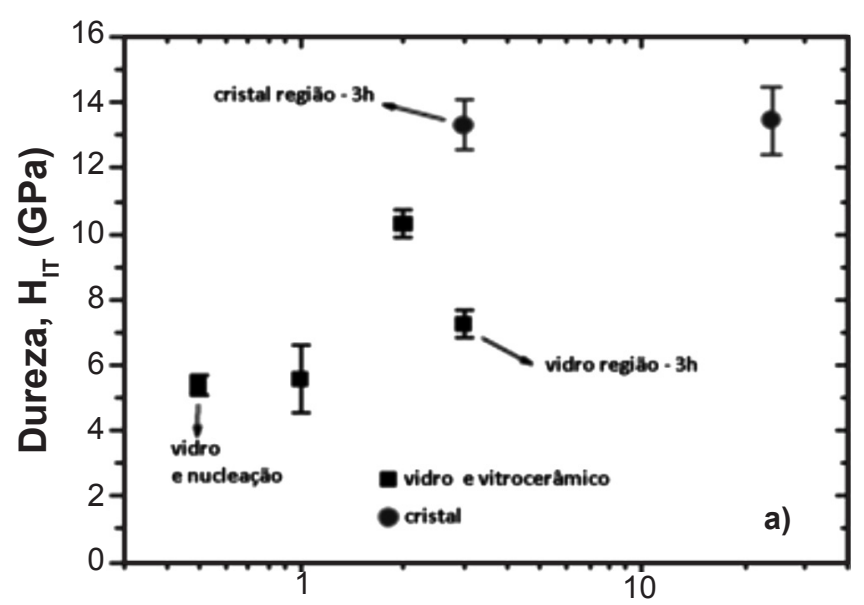

Tempo de tratamento térmico, $t(h)$ de nucleação e cristalização por $3 \mathrm{~h}$ a $563{ }^{\circ} \mathrm{C}$. A dureza e o módulo de elasticidade das duas diferentes matrizes apresentam maiores valores no cristal se comparados com as regiões que circundam o cristal (vidro), conforme indicado no gráfico (cristal região -3 h e vidro região -3 h). Foi usada uma carga baixa, de $20 \mathrm{mN}$, para não gerar trincas no cristal ou na região vítrea e também para que as deformações geradas por uma indentação não influenciassem a medida de dureza feita na indentação vizinha. Assim foi possível realizar várias indentações num só grão cristalino, sendo que as distâncias entre as indentações foram de $10 \mu \mathrm{m}$. Após as medidas de nanoindentação, as imagens foram feitas por microscopia óptica evidenciando as matrizes de indentações. A dureza das vitrocerâmicas é dependente da estrutura do cristal formado, da orientação cristalina e do estado de tensões residuais (tração ou compressão) em torno dos cristais. Os efeitos da anisotropia das estruturas sobre as medidas de dureza são bem conhecidos, porém os efeitos de tensão residual sobre a dureza são menos conhecidos. Nos materiais vitrocerâmicos, as tensões

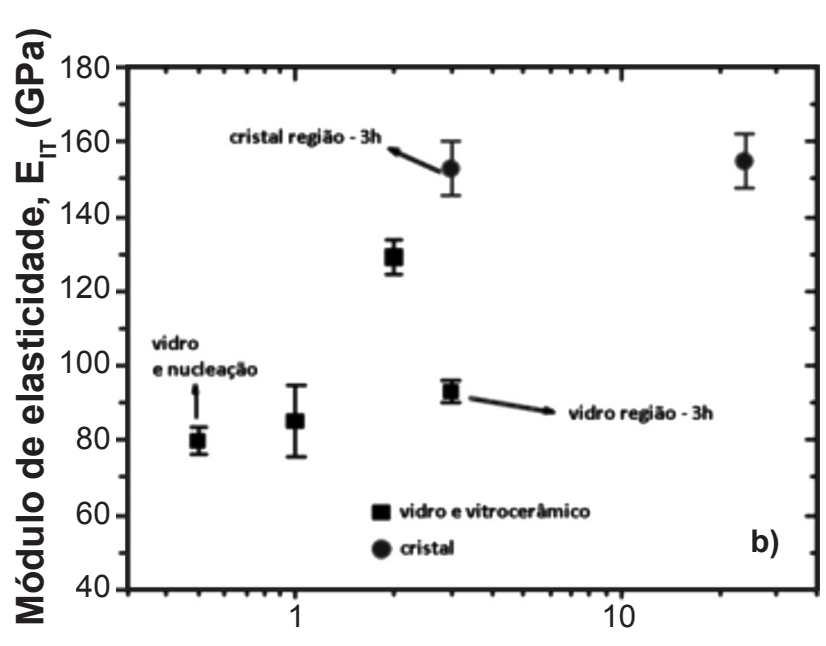

Tempo de tratamento térmico, $t(h)$

Figura 1: Gráficos da variação da dureza (a) e módulo de elasticidade (b) em função do tempo de tratamento térmico.

[Figure 1: Graphics of the variation of hardness (a) and modulus of elasticity $(b)$ as a function of the heat treatment time.]
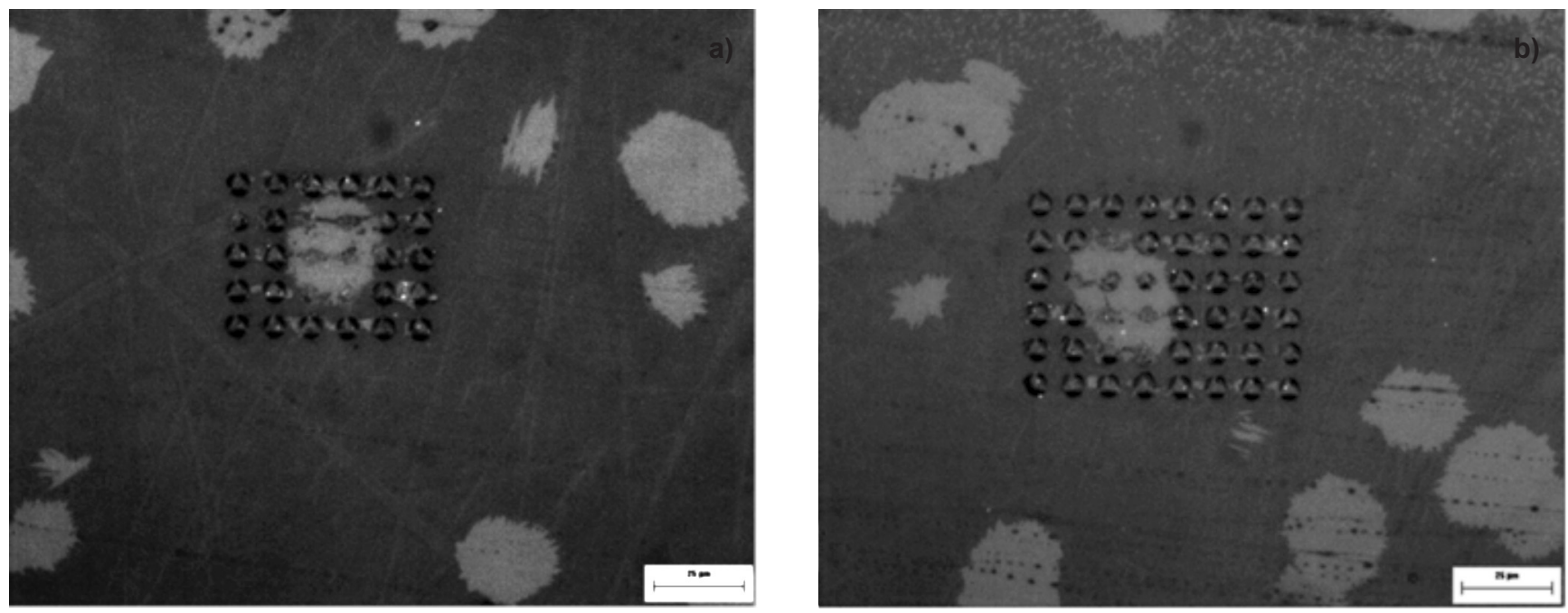

Figura 2: Imagens de microscopia óptica de duas diferentes matrizes de indentações em torno de cristais em amostras tratadas por $3 \mathrm{~h}$ a $563^{\circ} \mathrm{C}$. [Figure 2: Optical microscopy images of two different indentation matrices around crystals in the samples treated for $3 \mathrm{~h}$ at $\left.563{ }^{\circ} \mathrm{C}.\right]$ 
surgem durante o resfriamento da temperatura de transição vítrea para a temperatura ambiente, devido à diferença entre os coeficientes de expansão térmica entre as fases vítreas e cristalinas do LS . O coeficiente de expansão térmica do dissilicato de lítio para o vidro e cristal foi investigado. Os resultados mostraram que o coeficiente de expansão térmica do cristal é menor do que o do vidro, indicando que em temperatura ambiente os cristais embutidos na matriz vítrea estão sob compressão [23]. Em outro trabalho, foi utilizado a técnica de nanoindentação para medir o campo de tensões em torno de um cristal presente na matriz vítrea do dissilicato de lítio. Conforme mostrado, as propriedades mecânicas em torno de cristais embutidos na matriz vítrea são dependentes do estado de tensões em torno dos cristais [21]. No dissilicato de lítio parcialmente cristalizado, os cristais estão sob compressão e o campo de tensões agindo sobre a matriz vítrea é de tração circunferencial e compressão radial. O campo de tensões residuais em torno dos cristais é mais intenso numa região a distâncias menores que $100 \mu \mathrm{m}$ da superfície do cristal e diminui à medida que a distância aumenta. Em vitrocerâmicas de composição no sistema $\mathrm{MgO}-\mathrm{CaO}-\mathrm{SiO}_{2}-\mathrm{P}_{2} \mathrm{O}_{5}$, as tensões mecânicas são compressivas na superfície e crescem com o aumento da quantidade de $\mathrm{P}_{2} \mathrm{O}_{5}$ em relação a $\mathrm{SiO}_{2}$, o que provoca um aumento da tenacidade à fratura por indentação [9].

$\mathrm{Na}$ amostra tratada por $3 \mathrm{~h}$ existem dois comportamentos bem definidos: indentações na região do cristal e na região fora do cristal. Nesse caso, foi observado um comportamento bimodal. Nessa amostra, foi realizado um estudo mais detalhado das medidas de dureza, com um número maior de indentações. Como se pode ver nos gráficos da Fig. 3, existem dois grupos de valores de dureza e módulo elástico, que correspondem às regiões cristalinas e vítreas para essa amostra. Na amostra tratada por $3 \mathrm{~h}$ os cristais já possuem um tamanho suficientemente grande de modo que as indentações podem ser completamente feitas no interior de um só cristal. A região cristalina diferenciase da região vítrea pelos contornos de grãos (cristais) que são bem definidos. O contraste foi obtido posteriormente às medidas submetendo a amostra a um ataque químico. As amostras sofreram ataques químicos sucessivos com nital $2 \%$, com tempo de exposição de aproximadamente $4 \mathrm{~s}$, e entre cada novo ataque foi realizado um leve polimento com pasta de diamante de $0,25 \mu \mathrm{m}$, por aproximadamente $4 \mathrm{~s}$. O procedimento foi repetido até que a microestrutura revelasse nitidamente os cristais, tornando-os visíveis em um microscópio óptico, conforme mostrado na Fig. 2. Os resultados de módulo de elasticidade foram próximos aos encontrados anteriormente em [16], onde se determinou o módulo de elasticidade em função da cristalização. O módulo elástico foi $80 \pm 2 \mathrm{GPa}$ para o vidro e $133 \pm 5 \mathrm{GPa}$ para a vitrocerâmica cristalizada. Outros autores [28] também determinaram o módulo elástico de um vidro e de uma vitrocerâmica completamente cristalizada utilizando uma técnica ultrassônica. Obtiveram valores de 71 e 122 GPa para o vidro e a vitrocerâmica, respectivamente.

Comparação entre a dureza Knoop e Vickers: nas Figs.
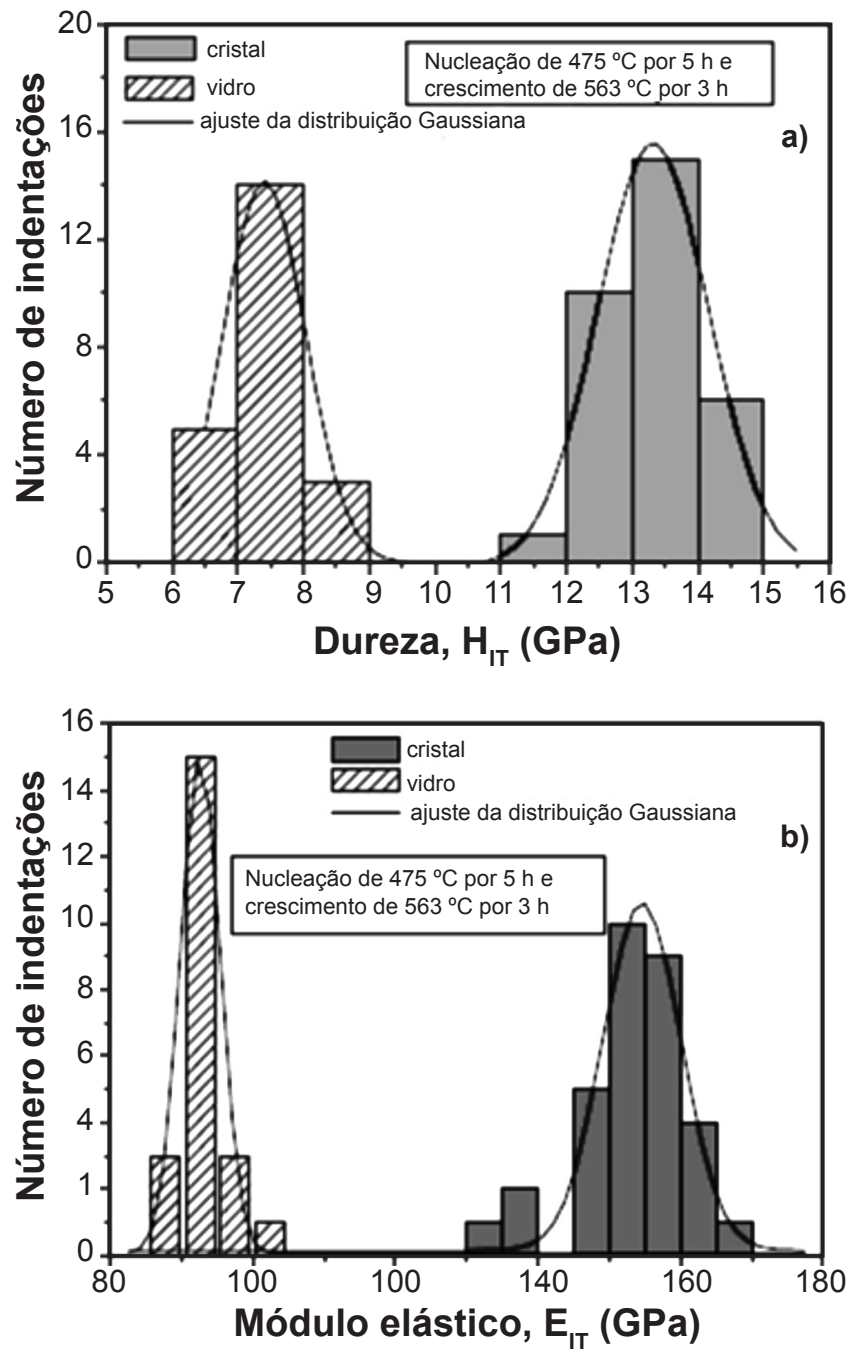

Figura 3: Gráficos de dois grupos de valores de dureza (a) e módulo de elasticidade (b) para uma amostra tratada a $475{ }^{\circ} \mathrm{C}$ por $5 \mathrm{~h}$ e crescimento de cristais a $563{ }^{\circ} \mathrm{C}$ por $3 \mathrm{~h}(73 \%$ de fração cristalina superficial).

[Figure 3: Graphs of two groups of hardness (a) and modulus of elasticity (b) values for a treated sample at $475^{\circ} \mathrm{C}$ for $5 \mathrm{~h}$ and crystal growth at $563{ }^{\circ} \mathrm{C}$ for $3 \mathrm{~h}$ (73\% of surface crystalline fraction).]

4a e 4b são comparados os resultados da microdureza Knoop e Vickers, respectivamente, com cargas aplicadas de 5 e $10 \mathrm{~N}$ em amostras tratadas por $5 \mathrm{~h}$ em $475^{\circ} \mathrm{C}$, em função do tempo de tratamento térmico a $563{ }^{\circ} \mathrm{C}$. Podese observar que as vitrocerâmicas de dissilicato de lítio possuem dureza maior para indentações com cargas menores. Os resultados estão de acordo com a literatura. Já foi estudada a dependência da carga de indentação sobre os valores de dureza para vidros alcalinos e verificado que a dureza decresce com o aumento da carga aplicada. Para a variação da dureza em função da carga de indentação, a dureza para o vidro solda-cal é observada decrescer com o aumento da carga aplicada. Portanto, a diminuição da dureza em função da carga aplicada observada para o dissilicato de lítio também é observada em outros tipos de vidros [29]. Em outro trabalho, foi determinada a dureza obtida a partir de indentação instrumentada em função da fração de volume cristalizada, levando a dureza a aumentar linearmente com 
a fração de volume cristalizada. A dureza do vidro foi de $6,5 \pm 0,2 \mathrm{GPa}$ e a do volume cristalizado foi de $8,3 \pm 0,6 \mathrm{GPa}$,
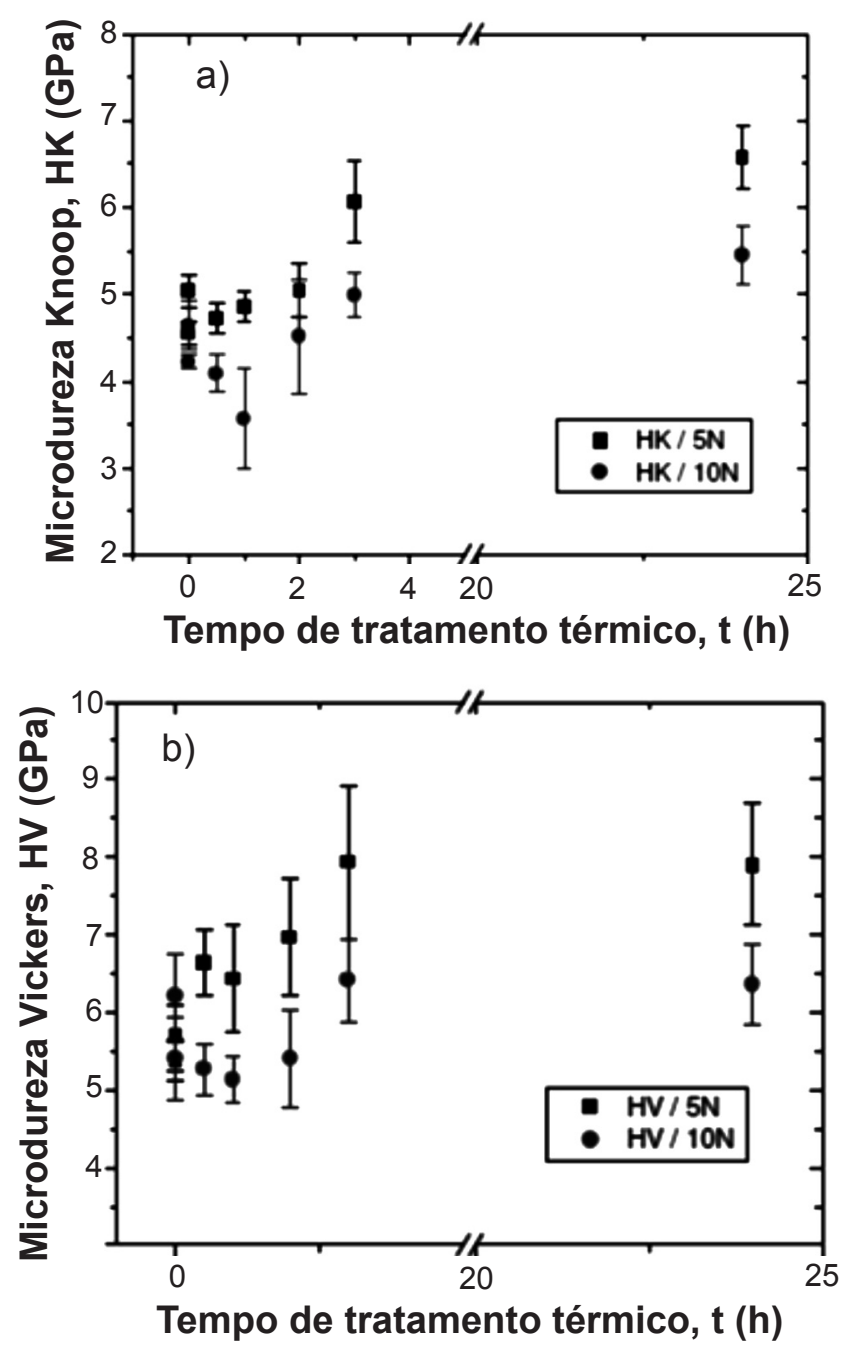

Figura 4: Variação da microdureza Knoop (a) e Vickers (b), com cargas de 5 e $10 \mathrm{~N}$ e para tempo de nucleação de $5 \mathrm{~h}$ a $475^{\circ} \mathrm{C}$, em função do tempo do tratamento térmico.

[Figure 4: Variation of Knoop (a) and (b) Vickers microhardness, with loads of 5 and $10 \mathrm{~N}$ and for nucleation time of $5 \mathrm{~h}$ at $475{ }^{\circ} \mathrm{C}$, as a function of the time of the heat treatment.]

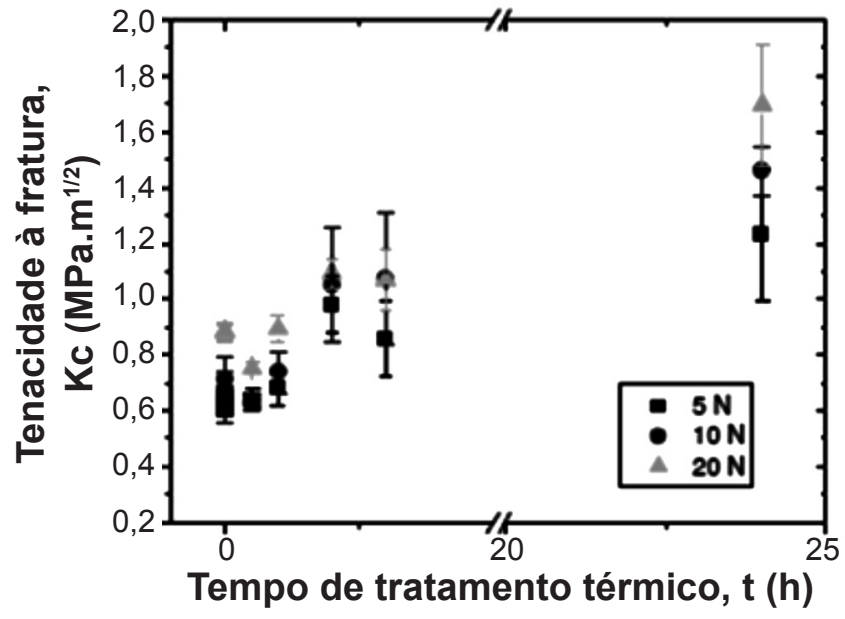

Figura 5: Tenacidade à fratura determinada a partir dos comprimentos de trinca induzida por indentação Vickers para cargas de 5, 10 e $20 \mathrm{~N}$ em função da fração cristalina.

[Figure 5: Fracture toughness determined from crack lengths induced by Vickers indentation for loads of 5, 10 and $20 \mathrm{~N}$ as a function of the crystalline fraction.]

mostrando um aumento de aproximadamente $28 \%$ [16].

Tenacidade à fratura por indentação: a Tabela I e a Fig. 5 resumem os resultados para amostras tratadas termicamente por diferentes tempos de crescimento de cristais. Observouse que a tenacidade à fratura por indentação aumentou com a fração cristalina independentemente da carga aplicada. Assim, os cristais agem como agentes de endurecimento na matriz vítrea, o que está de acordo com vários trabalhos na literatura [30-32]. Considerando o efeito da carga aplicada, até aproximadamente $80 \%$ de fração cristalina, não houve diferença significativa entre os valores de $\mathrm{K}_{\mathrm{C}}$ (Fig. 5), sendo considerável apenas para as amostras totalmente cristalinas. Este efeito ocorreu devido à dificuldade em medir os comprimentos das trincas radiais primárias em amostras parcialmente cristalinas, com baixas cargas aplicadas. Essa dificuldade reflete sobre os valores de $\mathrm{K}_{\mathrm{C}}$ para estas amostras. Mesmo a tenacidade à fratura por indentação com valor mais elevado obtido para vitrocerâmica totalmente cristalina com uma carga aplicada de $20 \mathrm{~N}$ é ainda inferior aos valores obtidos por testes padronizados de tenacidade à

Tabela I - Tenacidade à fratura por indentação e fração cristalina para diferentes tempos de tratamento térmico. [Table I - Fracture toughness by indentation and crystalline fraction for different heat treatment times.]

\begin{tabular}{cccccccc}
\hline $\begin{array}{c}\text { Tempo de } \\
\text { nucleação } \\
\left(475^{\circ} \mathrm{C}\right)\end{array}$ & $\begin{array}{c}\text { Tempo de } \\
\text { crescimento } \\
\left(563^{\circ} \mathrm{C}\right)\end{array}$ & $\begin{array}{c}\text { Fração } \\
\text { cristalina } \\
(\%)\end{array}$ & \multicolumn{2}{c}{$\begin{array}{c}\text { Tamanho médio dos cristais, } \\
\text { comprimento e largura }(\mu \mathrm{m})\end{array}$} & \multicolumn{3}{c}{$\begin{array}{c}\text { Tenacidade à fratura por } \\
\text { indentação }\left(\mathrm{MPa} . \mathrm{m}^{1 / 2}\right)\end{array}$} \\
\hline $0 \mathrm{~h}$ & $0 \mathrm{~h}$ & 0 & - & - & $0,6 \pm 0,0$ & $0,7 \pm 0,1$ & $0,8 \pm 0,0$ \\
$5 \mathrm{~h}$ & $0,5 \mathrm{~h}$ & 6 & 5,88 & 2,28 & $0,6 \pm 0,0$ & $0,7 \pm 0,0$ & $0,8 \pm 0,0$ \\
& $1 \mathrm{~h}$ & 15 & 12,40 & 6,66 & $0,7 \pm 0,1$ & $0,7 \pm 0,1$ & $0,9 \pm 0,1$ \\
& $2 \mathrm{~h}$ & 55 & 21,63 & 13,95 & $1,0 \pm 0,1$ & $1,1 \pm 0,2$ & $1,1 \pm 0,1$ \\
& $3 \mathrm{~h}$ & 73 & 43,02 & 22,62 & $1,0 \pm 0,1$ & $1,1 \pm 0,2$ & $1,1 \pm 0,1$ \\
& $24 \mathrm{~h}$ & $\sim 100$ & - & - & $1,2 \pm 0,2$ & $1,5 \pm 0,1$ & $1,7 \pm 0,2$ \\
\hline
\end{tabular}


fratura por indentação, como os encontrados em [32, 33]. A mesma constatação foi realizada em [16], em que os autores mostraram que a tenacidade à fratura por indentação apresentou uma dependência com a carga aplicada, a qual foi maior para a carga de $10 \mathrm{~N}$ do que para a carga de $5 \mathrm{~N}$. Outro trabalho [27] mostrou menor dependência da carga. O modelo foi aproximadamente $20 \%$ menor do que o modelo utilizado por Niihara. Também foi determinada a tenacidade à fratura por indentação pela técnica de dupla torção para a amostra de vidro, o qual foi de $0,75 \pm 0,01 \mathrm{MPa} . \mathrm{m}^{1 / 2}$, valor típico para vidros de silicato. A cristalização aumentou a

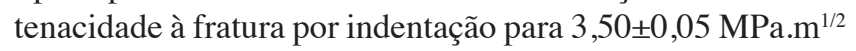
da amostra totalmente cristalizada, que foi aproximadamente cinco vezes maior que o valor para o vidro, mostrando que a dependência é quase linear [27].

Padrões de trincas: durante a penetração de uma ponta, geralmente de diamante, trincas podem ser geradas nos materiais, dependendo da forma do indentador e da carga aplicada. Cook e Pharr [34] analisaram e classificaram as trincas geradas por penetração em cinco tipos: cônica, radial, mediana, semicircular e lateral. Radial primária: são trincas que se propagam a partir dos cantos da impressão, com a mesma direção das diagonais da impressão, perpendicular à aplicação da carga. Radial secundária: são trincas que também se propagam a partir dos cantos da impressão, porém não possuem a mesma direção que a diagonal da impressão. Lateral: são trincas que se distribuem com direção paralela à superfície, em torno da impressão, perpendicular a direção de indentação. Trincas radiais e laterais apareceram com bastante frequência nas amostras analisadas. Na Fig. 6 são apresentados os padrões de trincas em função do tempo de tratamento térmico de crescimento de cristais. Verifica-se na Fig. $6 \mathrm{~b}$ a formação de trincas laterais em torno da impressão Vickers, o que não ocorreu na amostra tratada termicamente em 2 h, Fig. 6e. Maiores danos e maiores desvios das trincas radiais em torno dos cristais foram observados na amostra tratada por $3 \mathrm{~h}$ comparada com a amostra tratada por $2 \mathrm{~h}$. As trincas radiais tornam-se assim passíveis de erros de interpretação e podem ter contribuído para o decréscimo nos valores medidos de tenacidade à fratura por indentação. Com base na Fig. 6 pôde-se fazer algumas considerações sobre as mudanças apresentadas pelas trincas com a variação dos tratamentos térmicos (cristalização). Todas as indentações feitas com carga de $10 \mathrm{~N}$ apresentaram trincas radiais secundárias (Fig. 6). Observou-se que os vidros $\mathrm{LS}_{2}$ tratados por 24 e $3 \mathrm{~h}$ apresentaram o maior número delas. Uma análise realizada do processo de evolução das trincas radiais secundárias do vidro sem tratamento até a amostra tratada por 24 h é apresentada na Tabela II. Outra análise realizada foi da variação do número de trincas radiais secundárias no vidro sem tratamento e vidros com tratamentos térmicos de nucleação por $0,5,1,2,3$ e $24 \mathrm{~h}$. Em cada amostra foram analisadas 10 indentações com carga $10 \mathrm{~N}$ e os resultados de comprimento médio das trincas $(5$ e $10 \mathrm{~N})$, trincas radiais primárias, secundárias e laterais em função do tempo de tratamento térmico são apresentados na Tabela II.

Nos vidros $\mathrm{LS}_{2}$, tratados termicamente por 24 e $3 \mathrm{~h}$,
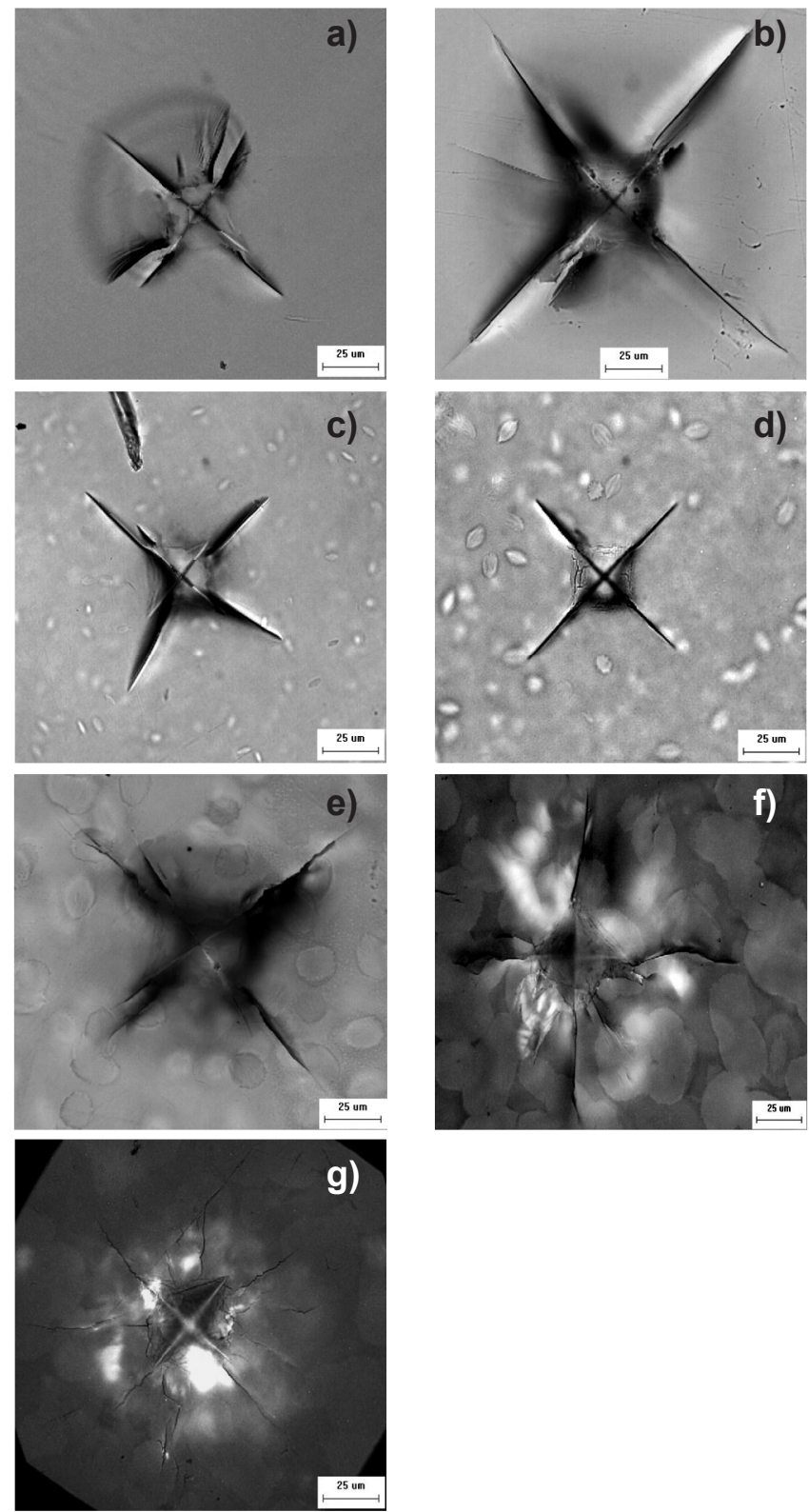

Figura 6: Imagens de microscopia óptica de indentações Vickers (carga $10 \mathrm{~N}$ ) realizadas em amostras de: a) vidro (fração cristalina, $\mathrm{FC}=0 \%)$; b) nucleadas $\left(475{ }^{\circ} \mathrm{C}\right.$ por $\left.2 \mathrm{~h} ; \mathrm{FC}=0 \%\right)+$ crescimento a $563{ }^{\circ} \mathrm{C}$ por: c) $0,5 \mathrm{~h}(\mathrm{FC}=6 \%)$; $) 1 \mathrm{~h}(\mathrm{FC}=15 \%)$; $) 2 \mathrm{~h}(\mathrm{FC}=55 \%)$; f) $3 \mathrm{~h}(\mathrm{FC}=73 \%)$; e g) $24 \mathrm{~h}(\mathrm{FC}=\sim 100 \%)$.

[Figure 6: Optical microscopy images of Vickers indentations (load $10 \mathrm{~N}$ ) performed on samples of: a) glass (crystalline fraction, $C F=0 \%)$; b) nucleated $\left(475{ }^{\circ} \mathrm{C}\right.$ for $\left.2 \mathrm{~h} ; \mathrm{CF}=0 \%\right)+$ growth at 563 ${ }^{\circ} \mathrm{C}$ for: c) $0.5 \mathrm{~h}(\mathrm{CF}=6 \%)$; d) $1 \mathrm{~h}(\mathrm{CF}=15 \%)$; e) $2 \mathrm{~h}(\mathrm{CF}=55 \%)$; $f$ ) $3 \mathrm{~h}(\mathrm{CF}=73 \%)$; and $\mathrm{g}) 2 \mathrm{~h}(\mathrm{CF}=\sim 100 \%)$.]

existiu em alguns casos uma grande imprecisão na medida da extensão da trinca radial primária devido ao grande número de trincas radiais secundárias produzidas pela indentação das cargas de 5 e $10 \mathrm{~N}$. Verificou-se algumas vezes que as trincas radiais não iniciaram preferencialmente nos cantos da impressão (Fig. 7), em que a concentração de tensão é alta, mas em algum ponto onde possivelmente existia um plano preferencial de clivagem. Quando se aumentou a 
Tabela II - Evolução das trincas radiais e número de trincas radiais.

[Table II - Evolution of radial cracks and number of radial cracks.]

\begin{tabular}{|c|c|c|c|}
\hline $\begin{array}{l}\text { Tempo de } \\
\text { tratamento térmico }\end{array}$ & $\begin{array}{l}\text { Padrões das trincas } \\
\text { (carga: } 5 \text { e } 10 \mathrm{~N} \text { ) }\end{array}$ & $\begin{array}{c}\text { Trincas radiais primárias e trincas radiais } \\
\text { secundárias }\end{array}$ & Trincas laterais \\
\hline$\underset{\rightarrow \text { nucleada }}{\mathrm{LS}_{2}}$ & $\begin{array}{l}\text { Pequena diferença entre o } \\
\text { comprimento da trinca radial } \\
\text { primária e secundária }\end{array}$ & $\begin{array}{l}\text { Aumento quase igual para carga de } 10 \text { e } 5 \mathrm{~N} \text { no } \\
\text { número de trincas radiais secundárias; carga de } \\
5 \mathrm{~N} \text { exibiu em torno da metade do aumento no } \\
\text { número de trincas radiais secundárias do que a } \\
\text { carga } 10 \mathrm{~N}\end{array}$ & $\begin{array}{l}\text { Todas as indentações } \\
\text { possuíram trincas } \\
\text { radiais primárias } \\
\text { nos quatro cantos da } \\
\text { impressão }\end{array}$ \\
\hline $\begin{array}{l}\text { Nucleada } \\
\rightarrow 0,5 \mathrm{~h}\end{array}$ & $\begin{array}{l}\text { Grande diferença entre o } \\
\text { comprimento da trinca radial } \\
\text { primária e secundária; a } \\
\text { trinca radial primária teve seu } \\
\text { comprimento reduzido quando } \\
\text { houve presença de trinca } \\
\text { radial secundária }\end{array}$ & $\begin{array}{c}\text { Decréscimo no número de trincas radiais } \\
\text { secundárias para carga de } 10 \text { e } 5 \mathrm{~N} \text {; o } \\
\text { decréscimo foi em torno de duas vezes maior } \\
\text { para carga de } 5 \mathrm{~N}\end{array}$ & $\begin{array}{l}\text { Foram observadas } \\
\text { trincas laterais em } \\
\text { todas as indentações }\end{array}$ \\
\hline $0,5 \mathrm{~h} \rightarrow 1 \mathrm{~h}$ & Decréscimo no comprimento & $\begin{array}{l}\text { Decréscimo no número de trincas radiais } \\
\text { secundárias para carga } 5 \text { e } 10 \mathrm{~N}\end{array}$ & $\begin{array}{l}\text { Foram observadas } \\
\text { trincas laterais em } \\
\text { todas as indentações }\end{array}$ \\
\hline $1 \mathrm{~h} \rightarrow 2 \mathrm{~h}$ & Decréscimo no comprimento & $\begin{array}{l}\text { Decréscimo no número de trincas radiais } \\
\text { secundárias para carga } 5 \text { e } 10 \mathrm{~N}\end{array}$ & $\begin{array}{l}\text { Foram observadas } \\
\text { trincas laterais em } \\
\text { todas as indentações }\end{array}$ \\
\hline $2 \mathrm{~h} \rightarrow 3 \mathrm{~h}$ & $\begin{array}{l}\text { Decréscimo no comprimento } \\
\text { médio das trincas radiais } \\
(10 \mathrm{~N}) ; \text { quase igualdade no } \\
\text { comprimento médio das } \\
\text { trincas radiais }(5 \mathrm{~N})\end{array}$ & $\begin{array}{l}\text { Grande salto no número de trincas radiais } \\
\text { secundárias; em particular, a carga de } 10 \mathrm{~N} \\
\text { registrou um aumento de três vezes no número } \\
\text { de trincas radiais secundárias; carga de } 5 \mathrm{~N} \\
\text { registrou um aumento maior no número de } \\
\text { trincas radiais secundárias, do que a carga } 10 \mathrm{~N}\end{array}$ & $\begin{array}{l}\text { Foram observadas } \\
\text { trincas laterais em } \\
\text { todas as indentações }\end{array}$ \\
\hline $3 \mathrm{~h} \rightarrow 24 \mathrm{~h}$ & Decréscimo no comprimento & $\begin{array}{l}\text { O número de trincas radiais secundárias teve } \\
\text { um grande salto; o salto foi menor em } 3 \mathrm{~h} \text { do } \\
\text { que } 24 \mathrm{~h} \text {; decréscimo variando de } 7 \% \text { a } 8 \% \text { no } \\
\text { comprimento médio das trincas radiais }\end{array}$ & $\begin{array}{l}\text { Foram observadas } \\
\text { trincas laterais em } \\
\text { todas as indentações }\end{array}$ \\
\hline
\end{tabular}
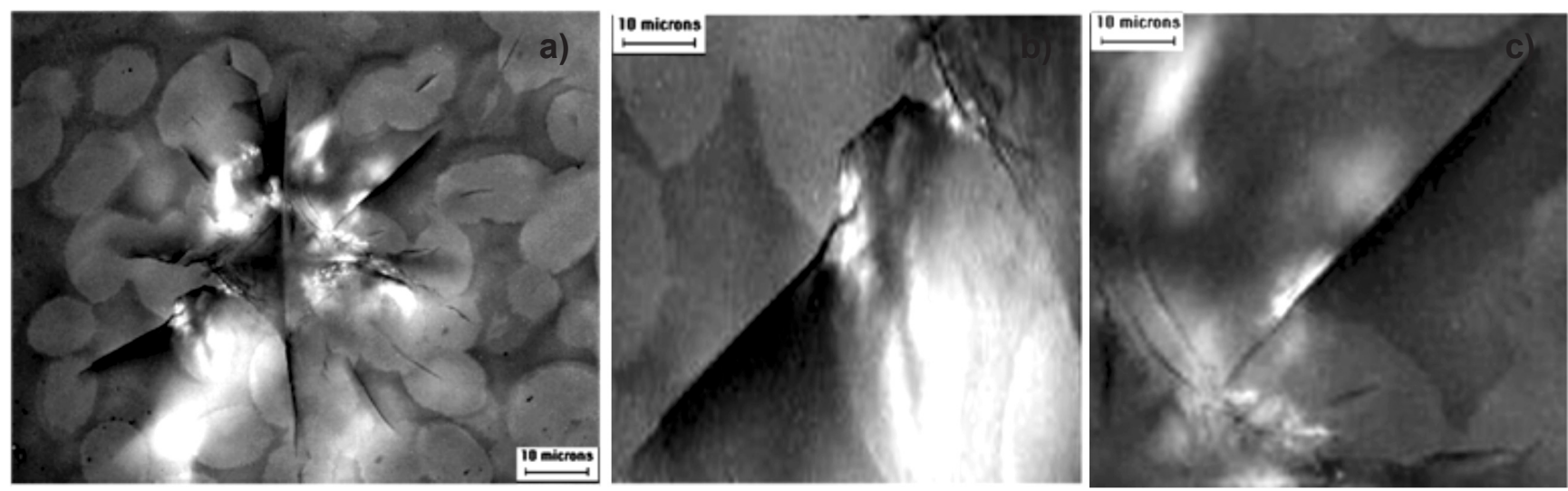

Figura 7: Imagens obtidas por microscopia óptica mostrando detalhes de trinca radial que não se propagou a partir do canto da impressão (b, c) em uma indentação Vickers com carga de $10 \mathrm{~N}$ (a) em vidro dissilicato de lítio tratado a $3 \mathrm{~h}$ a $563^{\circ} \mathrm{C}$.

[Figure 7: Images obtained by light microscopy showing details of radial crack that did not propagate from the corner of the impression (b, c) in a Vickers indentation with $10 \mathrm{~N}$ load (a) on treated lithium disilicate glass for 3 h at $563{ }^{\circ} \mathrm{C}$.]

fração cristalina superficial houve maior influência da formação das trincas radiais secundárias e laterais. Sendo assim, quando se aumenta a cristalização, os planos de fratura na direção da diagonal da impressão podem não ser pontos preferenciais de propagação das trincas. Na literatura é mostrada a dependência da tenacidade à fratura por indentação com a orientação cristalográfica. A medida do comprimento das trincas radiais foi utilizada para determinar a tenacidade à fratura por indentação. Foi verificado que as trincas geralmente não se propagam a partir dos cantos da impressão. Tais trincas claramente propagam-se ao longo dos planos preferenciais de clivagem. A tenacidade à fratura por indentação de $\mathrm{SiC}$ é comparativamente mais baixa ao longo destes planos. A tenacidade à fratura por 
indentação obtida foi de 1,84 MPa.m ${ }^{1 / 2}$ no caso em que as trincas se propagaram na direção dos planos preferenciais de clivagem, enquanto que o valor de 3,46 MPa.m ${ }^{1 / 2}$ foi observado quando as trincas ocorreram na mesma direção da diagonal da impressão [13]. Em outro trabalho, os autores mostram imagens de indentações Vickers com carga $10 \mathrm{~N}$ nas amostras de $\mathrm{LS}_{2}$ parcialmente cristalizadas em 3, 12, 33 e $84 \%$. À medida que a fração de volume cristalizado aumenta, as trincas mudam sua direção de propagação, seguindo os caminhos mais favoráveis [16]. Assim, como na vitrocerâmica, a impressão atinge diferentes grãos cristalinos e é de se esperar uma grande dispersão nos padrões de crescimento de trincas. As medidas de tenacidade à fratura por indentação apresentaram valores maiores para cargas altas em todas as frações cristalinas. Para a amostra com fração cristalina superficial de $100 \%$, a carga de $5 \mathrm{~N}$ apresentou resultado intermediário entre os obtidos para 10 e $20 \mathrm{~N}$ e com menor dispersão nas medidas. Para a amostra com fração cristalina superficial de $73 \%$ as cargas de $20 \mathrm{e}$ $10 \mathrm{~N}$ apresentaram resultados semelhantes. No entanto, com carga de $20 \mathrm{~N}$ houve menor dispersão nos resultados. Desta forma, pode-se dizer que para "regiões cristalinas" a carga de $10 \mathrm{~N}$ (intermediária) forneceu melhores resultados de tenacidade à fratura por indentação. Comparando amostras com $100 \%$ de fase cristalina a amostra do dissilicato de lítio com tratamento térmico na condição de nucleação e crescimento por $3 \mathrm{~h}$ a $563{ }^{\circ} \mathrm{C}$ apresentou maiores valores de tenacidade à fratura por indentação. Um tempo maior de tratamento térmico de nucleação garante a formação de um maior número de cristais, o que resulta em maiores valores de tenacidade à fratura por indentação.

\section{CONCLUSÕES}

Dissilicato de lítio amorfo apresentou dureza de 5,18 $\mathrm{GPa}$, módulo de elasticidade $80 \mathrm{GPa}$ e tenacidade à fratura por indentação de $0,8 \mathrm{MPa} \cdot \mathrm{m}^{1 / 2}$. Amostras com alta fração cristalina apresentaram valores de dureza, módulo de elasticidade e tenacidade à fratura por indentação com pequenas variações, sendo microdureza Knoop de 7,2 GPa, módulo de elasticidade de $154,8 \mathrm{GPa}$ e tenacidade à fratura por indentação de 1,8 MPa.m ${ }^{1 / 2}$. Durezas medidas usando penetrador Vickers foram sempre maiores que os obtidos usando penetrador Knoop. Este resultado é semelhante ao obtido na literatura para testes em materiais cerâmicos. Houve uma diminuição da dureza em função da carga aplicada obtida para o dissilicato de lítio, que também é observada em outros tipos de vidros. Com o aumento da fração cristalina superficial, a tenacidade aumentou em relação ao vidro dissilicato de lítio sem tratamento térmico. Para o tratamento térmico de $24 \mathrm{~h}$, ocorreu um grande acréscimo no valor de tenacidade à fratura por indentação. Com a cristalização, ocorreu uma modificação no tipo de formação e propagação das trincas relacionada com a energia necessária para a formação da trinca radial. No vidro e nas vitrocerâmicas com baixa fração cristalina, as trincas radiais primárias que apareceram após a penetração Vickers foram mais bem definidas, com poucas trincas radiais secundárias ou trincas laterais. Para altas frações cristalinas, a extensão das trincas radiais primárias foi menor e apareceram trincas radiais secundárias e trincas laterais em grande número.

\section{AGRADECIMENTOS}

Os autores agradecem à Coordenação de Aperfeiçoamento de Pessoal do Nível Superior (CAPES) e ao Conselho Nacional de Desenvolvimento Científico e Tecnológico (CNPq) pelo apoio financeiro e ao Laboratório de Materiais Vítreos (LAMAV) da Universidade Federal de São Carlos (UFSCar) pelo fornecimento do material para a pesquisa.

\section{REFERÊNCIAS}

[1] B.R. Lawn, Fracture of brittle solids, Cambridge Un. Press, New York (1995).

[2] W.D. Callister, Ciência e engenharia de materiais: uma introdução, $5^{\text {a }}$ Ed., LTC, Rio de Janeiro (2002).

[3] R.G. Munro, "Data evaluation theory and practice for materials properties", Spec. Publ. 960-11, NIST, Gaithersburg (2003) 79.

[4] A. Şakar-Deliormanli, M. Güden, J. Biomed. Mater. Res. B Appl. Biomater. 76 (2005) 257.

[5] F. Sergejev, M. Antonov, Proc. Eston. Acad. Sci. Eng. 12 (2006) 388.

[6] C.B. Ponton, R.D. Rawlings, Mater. Sci. Tech. 5 (1989) 865.

[7] J.J. Kruzic, R.O. Ritchie, J. Am. Ceram. Soc. 86 (2003) 1433.

[8] P.W. McMillan, Glass ceramics, $2^{\text {nd }}$ Ed., Academic Press, New York (1979)

[9] V.K. Marghussian, A.S.M. Mesgar, Ceram. Int. 26 (2000) 415.

[10] A. Clifford, R. Hill, J. Non-Cryst. Solids 196 (1996) 346.

[11] S. Morimoto, E. Waraporn, J. Ceram. Soc. Jpn. 112 (2004) 259.

[12] W. Zheng, J. Cheng, X. Lou, J. Liu, Trans. Nonferrous Met. Soc. China 16 (2006) 105.

[13] G.M. Pharr, Y. Katoh, H. Bel, J. Undergrad. Res. 9 (2003) 59.

[14] S. Huang, P. Caoa, C. Wanga, Z. Huang, W. Gaoa, J. Asian Ceram. Soc. 1 (2013) 46.

[15] T. Zhao, Y. Qin, P. Zhang, B. Wang, J. Yangn, Ceram. Int. 40 (2014) 12449.

[16] F.C. Serbena, I. Mathias, C.E. Foerstera, E.D. Zanotto, Acta Mater. 86 (2015) 216.

[17] E. Apel, J. Deubener, A. Bernard, M. Höland, R. Müller, H. Kappert, V. Rheinberger, W. Höland, J. Mech. Behav. Biomed. Mater. 1 (2008) 313.

[18] S. Buchner, C.M. Lepienski, P.C. Soares Jr, N.M. Balzaretti, Mater. Sci. Eng. A 528 (2011) 3921.

[19] A. Alao, L. Yinn, J. Mech. Behav. Biomed. Mater. 49 (2015) 162.

[20] S. Buchner, C. Radtke, N.M. Balzaretti, J. Inorg. Non- 
Met. Mater. 3 (2013) 15.

[21] P.C. Soares Jr, C.M. Lepienski, J. Non-Cryst. Solids 348 (2004) 139.

[22] V.R. Mastelaro, E.D. Zanotto, J. Non-Cryst. Solids 194 (1996) 297.

[23] V.R. Mastelaro, E.D. Zanotto, J. Non-Cryst. Solids 247 (1999) 79.

[24] H. Pinto, L. Ito, M. Crovace, E.B. Ferreira, F. Fauth, T. Wroblewski, E.D. Zanotto, A.R. Pyzalla, J. Non-Cryst. Solids 353 (2007) 207.

[25] D.G. Quinn, C.R Bradt, J. Am. Ceram. Soc. 90, 3 (2007) 673.

[26] W.C. Oliver, G.M. Pharr, J. Mater. Res. 7 (1992) 1564. [27] G.R. Anstis, P. Chantikul, B.R. Lawn, D.B. Marshall, J.
Am. Ceram. Soc. 64 (1981) 533.

[28] Z. Strnad, Glass-ceramic materials: glass science and technology, Elsevier Publ., New York (1986) 286.

[29] J. Gong, W. Si, Z. Guan, J. Non-Cryst. Solids 282 (2001)325.

[30] J.M. Rincon, F. Capel. Ceram. Intern. 11, 3 (1985) 97.

[31] K.J. Anusavice, N. Zhang, J. Am. Ceram. Soc. 80 (1997) 1353.

[32] N. Zhang, K.J. Anusavice, J. Am. Ceram. Soc. 82 (1999) 2509.

[33] M. Albakry, M. Guazzato, M.V. Swain, J. Dent. 31 (2003) 181.

[34] R.F. Cook, G.M. Pharr, J. Am. Ceram. Soc. 73 (1990) 787.

(Rec. 26/06/2017, Rev.08/09/2017, 04/11/2017, 06/12/2017, Ac. 07/12/2017) 